APS/1: Thin-shell-wormhole

\title{
Thin-shell wormholes from charged black holes in generalized dilaton-axion gravity
}

\author{
A. A. Usman * and Z. Hasan* \\ Department of Physics, Aligarh Muslim University, Aligarh 202 002, Uttar Pradesh, India. \\ F. Rahaman丹 and Sk. A. Rakil讯 \\ Department of Mathematics, Jadavpur University, Kolkata 700 032, West Bengal, India \\ Saibal Ray \\ Department of Physics, Government College of Engineering 83 Ceramic Technology, Kolkata 700 010, West Bengal, India \\ Peter K. F. Kuhfittig \\ Department of Mathematics, Milwaukee School of Engineering, Milwaukee, Wisconsin 53202-3109, USA
}

(Dated: November 12, 2018)

\begin{abstract}
This paper discusses a new type of thin-shell wormhole constructed by applying the cut-and-paste technique to two copies of a charged black hole in generalized dilaton-axion gravity, which was inspired by low-energy string theory. After analyzing various aspects of this thin-shell wormhole, we discuss its stability to linearized spherically symmetric perturbations.
\end{abstract}

PACS numbers: 95.30.Sf, 95.36.+x, 04.20.Jb

\section{INTRODUCTION}

The study of traversable wormholes has received considerable attention from researchers for the past two decades. Although lacking observational evidence, wormholes are just as good a prediction of the general theory of relativity as black holes. In particular, we refer to the pioneering work of Visser [1], who proposed a theoretical method for constructing a new class of traversable Lorentzian wormholes from black-hole spacetimes. This construction proceeds by surgically grafting two Schwarzschild spacetimes together in such a way that no event horizon is permitted to form. The resulting structure is a wormhole spacetime in which the throat is a three-dimensional thin shell. In recent years, Visser's approach was adopted by various authors for constructing thin-shell wormholes by similar methods, generally requiring spherical symmetry $[2,3,4,5,6,7,6,9,10$, $11,12,13,14,15,16,17,18,19,20]$. The approach is of special interest because it minimizes the amount of exotic matter required. All the exotic matter is confined to the shell.

More recently, Sur, Das, and SenGupta 21] discovered a new black-hole solution for Einstein-Maxwell scalar field systems inspired by low-energy string theory. They considered a generalized action in which two scalar fields are minimally coupled to an Einstein-Hilbert-Maxwell

\footnotetext{
*Electronic address: anisul@iucaa.ernet.in

${ }^{\dagger}$ Electronic address: farook_rahaman@yahoo.com

${ }^{\ddagger}$ Electronic address: saibal@iucaa.ernet.in

$\S$ Electronic address: kuhfitti@msoe.edu
}

field in four dimensions,

$$
I=\frac{1}{2 \kappa} \int d^{4} x \sqrt{-g}\left[R-\frac{1}{2} \partial_{\mu} \varphi \partial^{\mu} \varphi-W\right],
$$

where

$$
\begin{array}{r}
W=\frac{1}{2} \omega(\varphi) \partial_{\mu} \zeta \partial^{\mu} \zeta-\alpha(\varphi, \zeta) F_{\mu \nu} F^{\mu \nu} \\
-\beta(\varphi, \zeta) F_{\mu \nu} F^{\mu \nu *}
\end{array}
$$

$\kappa=8 \pi G, R$ is the curvature scalar, $F_{\mu \nu}$ is the Maxwell field tensor, while $\varphi$ and $\zeta$ are two massless scalar or pseudo scalar fields, which are coupled to the Maxwell field. This coupling is described by the functions $\alpha$ and $\beta$. Here $\zeta$ acquires a non-minimal kinetic term $\omega$. In the context of low-energy string theory, fields $\phi$ and $\xi$ can be identified as massless scalar dilaton and pseudo scalar axion fields, respectively.

With the above action, Eq. (1), Sur, et al., 21] found the most general class of black-hole solutions and obtained two types of metrics, classified as asymptotically flat and asymptotically non-flat. Since we are interested in obtaining a thin-shell wormhole from this new black hole, we adopt the asymptotically flat metric given by

$$
d s^{2}=-f(r) d t^{2}+f(r)^{-1} d r^{2}+h(r)\left(d \theta^{2}+\sin ^{2} \theta d \phi^{2}\right),
$$

where

$$
f(r)=\frac{\left(r-r_{-}\right)\left(r-r_{+}\right)}{\left(r-r_{0}\right)^{2-2 n}\left(r+r_{0}\right)^{2 n}},
$$

and

$$
h(r)=\frac{\left(r+r_{0}\right)^{2 n}}{\left(r-r_{0}\right)^{2 n-2}},
$$

where, according to Ref. [21], the exponent $n$ is a dimensionless constant stricly greater than 0 and stricly less 
than 1. In addition, various other parameters are given by

$$
\begin{aligned}
r_{ \pm} & =m_{0} \pm \sqrt{m_{0}^{2}+r_{0}^{2}-\frac{1}{8}\left(\frac{K_{1}}{n}+\frac{K_{2}}{1-n}\right)}, \\
r_{0} & =\frac{1}{16 m_{0}}\left(\frac{K_{1}}{n}-\frac{K_{2}}{1-n}\right) \\
m_{0} & =m-(2 n-1) r_{0}, \\
K_{1} & =4 n\left[4 r_{0}^{2}+2 r_{0}\left(r_{+}+r_{-}\right)+r_{+} r_{-}\right], \\
K_{2} & =4(1-n) r_{+} r_{-}, 0<n<1, \\
m & =\frac{1}{16 r_{0}}\left(\frac{K_{1}}{n}-\frac{K_{2}}{1-n}\right)+(2 n-1) r_{0},
\end{aligned}
$$

where $m$ is the mass of the black hole. The parameters $r_{+}$and $r_{-}$are the inner and outer event horizons, respectively. Also, $r=r_{0}$ is a curvature singularity; the parameters obey the condition $r_{0}<r_{-}<r_{+}$.

In this paper we present a new kind of thin-shell wormhole by surgically grafting two charged black holes in generalized dilaton-axion gravity. The exotic matter required for its physical existence may possibly be collected from scalar fields that built the black holes. Various aspects of this thin-shell wormhole are analyzed, particularly the equation of state relating pressure and density. Also discussed is the attractive or repulsive nature of the wormhole, as well as the energy conditions on the shell. Our final topic is a stability analysis to determine the conditions under which the wormhole is stable to linearized radial perturbations. A comparison to the stability of other thin-shell wormholes in the literature is also made.

\section{THIN-SHELL WORMHOLE CONSTRUCTION}

The mathematical construction of our thin-shell wormhole begins by taking two copies of the black hole and removing from each the four-dimensional region

$$
\Omega^{ \pm}=\left\{r \leq a \mid a>r_{+}\right\}
$$

We now identify (in the sense of topology) the timelike hypersurfaces

$$
\partial \Omega^{ \pm}=\left\{r=a \mid a>r_{+}\right\}
$$

denoted by $\Sigma$. The resulting manifold is geodesically complete and consists of two asymptotically flat regions connected by a throat. The induced metric on $\Sigma$ is given by

$$
d s^{2}=-d \tau^{2}+a(\tau)^{2}\left(d \theta^{2}+\sin ^{2} \theta d \phi^{2}\right),
$$

where $\tau$ is the proper time on the junction surface. Using the Lanczos equations 1, 2, 3, 4, 5, 6, 7, 8, 9, 10, 11, 12, $13,14,15,16,17,18,19,20]$, one can obtain the surface stress energy tensor $S_{j}^{i}=\operatorname{diag}\left(-\sigma, p_{\theta}, p_{\phi}\right)$, where $\sigma$ is the surface energy density and $p_{\theta}$ and $p_{\phi}$ are the surface pressures. The Lanczos equations now yield [7]

$$
\sigma=-\frac{1}{4 \pi} \frac{h^{\prime}(a)}{h(a)} \sqrt{f(a)+\dot{a}^{2}}
$$

and

$$
p_{\theta}=p_{\phi}=p=\frac{1}{8 \pi} \frac{h^{\prime}(a)}{h(a)} \sqrt{f(a)+\dot{a}^{2}}+\frac{1}{8 \pi} \frac{2 \ddot{a}+f^{\prime}(a)}{\sqrt{f(a)+\dot{a}^{2}}} .
$$

To understand the dynamics of the wormhole, we assume the radius of the throat to be a function of proper time, or $a=a(\tau)$. Also, overdot and prime denote, respectively, the derivatives with respect to $\tau$ and $a$. For a static configuration of radius $a$, we obtain the respective values of the surface energy density and the surface pressures. For a static configeration of radius $a$, we obtain (assuming $\dot{a}=0$ and $\ddot{a}=0$ ) from Eqs. (12) and (13),

$$
\sigma=-\frac{4\left[a+(1-2 n) r_{0}\right]}{D} \frac{\left(a-r_{-}\right)\left(a-r_{+}\right)}{\left(a-r_{0}\right)\left(a+r_{0}\right)}
$$

and

$$
p_{\theta}=p_{\phi}=p=\frac{2 a-r_{-}-r_{+}}{D}
$$

where

$$
D=8 \pi\left(a-r_{0}\right)^{1-n}\left(a+r_{0}\right)^{n} \sqrt{\left(a-r_{-}\right)\left(a-r_{+}\right)} .
$$

Observe that the energy density $\sigma$ is negative. The pressure $p$ may be positive, however. This would depend on the position of the throat and hence on the physical parameters $r_{0}, r_{-}$, and $r_{+}$defining the wormhole. Similarly, $p+\sigma, \sigma+2 p$, and $\sigma+3 p$, obtained by using the above equations, may also be positive under certain conditions, in which case the strong energy condition is satisfied.

Keeping in mind the condition $r_{+}>r_{-}>r_{0}$ for different radii defining the wormhole, we plot $p$ versus $a$ in Fig. 1. We choose typical wormholes whose radii $\left(r_{0}, r_{-}\right.$, and $r_{+}$) fall within the range 2 to $12 \mathrm{kms}$. Also taken into account is the sensitivity of the plots with respect to $n$, as described in the caption of the figure.

\section{THE GRAVITATIONAL FIELD}

We now turn our attention to the attractive or repulsive nature of our wormhole. To perform the analysis, we calculate the observer's four-acceleration $a^{\mu}=u_{; \nu}^{\mu} u^{\nu}$, where $u^{\nu}=d x^{\nu} / d \tau=(1 / \sqrt{f(r)}, 0,0,0)$. In view of the line element, Eq. (2), the only non-zero component is given by

$$
a^{r}=\Gamma_{t t}^{r}\left(\frac{d t}{d \tau}\right)^{2}=\frac{1}{2} \frac{A r^{2}-B r+C}{\left(r-r_{0}\right)^{3-2 n}\left(r+r_{0}\right)^{2 n+1}}
$$




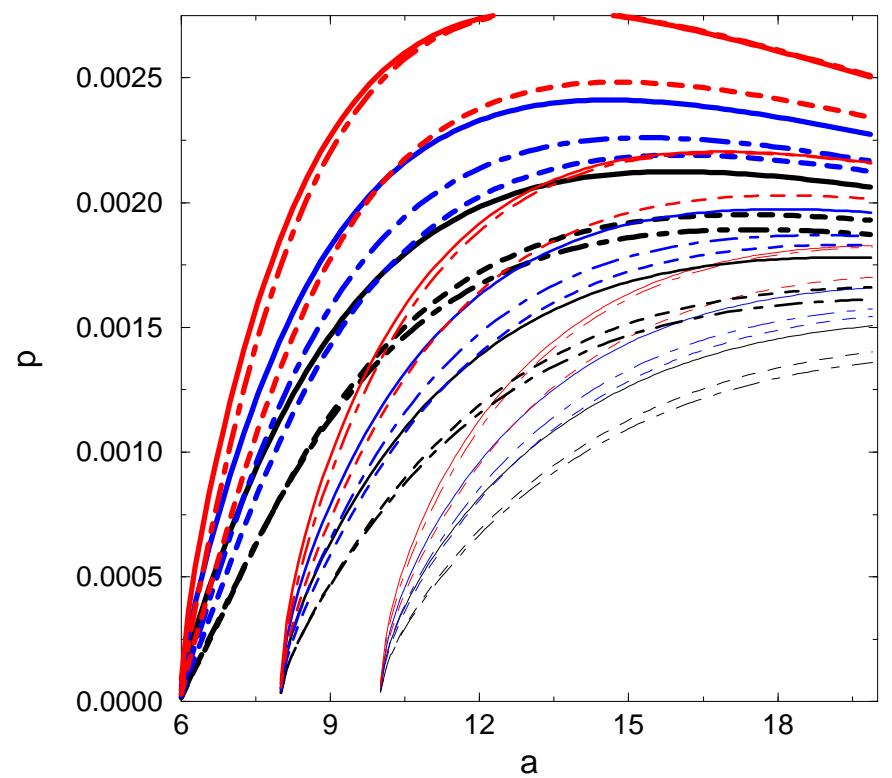

FIG. 1: Plot for $p$ versus $a$. The black, blue, and red colors represent $n=0.98,0.5$ and 0.02 , respectively. For every color, thin, thick, and thicker curves, respectively, represent $r_{+}=$ 10,8 , and 6 . For every combination of $r_{+}$and $n$, we plot three different sets, $\left(r_{-}=5, r_{0}=2\right),\left(r_{-}=5, r_{0}=3\right)$, and $\left(r_{-}=4, r_{0}=2\right)$, which are represented by chain and solid curves, respectively.

where,

$$
\begin{gathered}
A=r_{-}+r_{+}+4 n r_{0}-2 r_{0}, \\
B=2 r_{0}^{2}+\left(r_{-}+r_{+}\right)\left(4 n r_{0}-2 r_{0}\right)+2 r_{-} r_{+},
\end{gathered}
$$

and

$$
C=r_{0}^{2}\left(r_{-}+r_{+}\right)+\left(4 n r_{0}-2 r_{0}\right) r_{-} r_{+} .
$$

A radially moving test particle initially at rest obeys the equation of motion

$$
\frac{d^{2} r}{d \tau^{2}}=-\Gamma_{t t}^{r}\left(\frac{d t}{d \tau}\right)^{2}=-a^{r}
$$

If $a^{r}=0$, we obtain the geodesic equation. Moreover, a wormhole is attractive if $a^{r}>0$ and repulsive if $a^{r}<0$. These characteristics depend on the parameters $r_{0}, r_{-}$, $r_{+}$, and $n$, the conditions on which can be conveniently expressed in terms of the coefficients $A, B$, and $C$. To avoid negative values for $r$, let us consider only the root $r=\left(B+\sqrt{B^{2}-4 A C}\right) /(2 A)$ of the quadratic equation $A r^{2}-B r+C=0$. It now follows from Eq. (17) that $a^{r}=0$ whenever

$$
\left(r-\frac{B}{2 A}\right)^{2}=\frac{B^{2}-4 A C}{4 A^{2}} .
$$

For the attractive case, $a^{r}>0$, the condition becomes

$$
\left(r-\frac{B}{2 A}\right)^{2}>\frac{B^{2}-4 A C}{4 A^{2}}
$$

For the repulsive case, $a^{r}<0$, the sense of the inequality is reversed.

\section{THE TOTAL AMOUNT OF EXOTIC MATTER}

In this section we determine the total amount of exotic matter for the thin-shell wormhole. This total can be quantified by the integral [7, 8, 9, 10, 11, 12, 13]

$$
\Omega_{\sigma}=\int[\rho+p] \sqrt{-g} d^{3} x
$$

By introducing the radial coordinate $R=r-a$, w get

$$
\Omega_{\sigma}=\int_{0}^{2 \pi} \int_{0}^{\pi} \int_{-\infty}^{\infty}[\rho+p] \sqrt{-g} d R d \theta d \phi
$$

Since the shell is infinitely thin, it does not exert any radial pressure. Moreover, $\rho=\delta(R) \sigma(a)$. So

$$
\begin{aligned}
& \Omega_{\sigma}=\left.\int_{0}^{2 \pi} \int_{0}^{\pi}[\rho \sqrt{-g}]\right|_{r=a} d \theta d \phi=4 \pi h(a) \sigma(a) \\
= & -\frac{16 \pi\left[a+(1-2 n) r_{0}\right]}{D}\left[\frac{\left(a-r_{-}\right)\left(a-r_{+}\right)}{\left(a-r_{0}\right)^{2 n-1}\left(a+r_{0}\right)^{1-2 n}}\right] .
\end{aligned}
$$

Here $D$ is given in Eq. (16).

This NEC violating matter can be reduced by taking the value of $a$ closer to $r_{+}$, the location of the outer event horizon. The closer $a$ is to $r_{+}$, however, the closer the wormhole is to a black hole: incoming microwave background radiation would get blueshifted to an extremely high temperature [22]. On the other hand, it follows from Eq. (20) that for $a \gg r_{+}, \Omega_{\sigma}$ will depend linearly on $a$ :

$$
\Omega_{\sigma} \approx-2 a
$$

\section{AN EQUATION OF STATE}

Taking the form of the equation of state (EoS) to be $p=w \sigma$, we obtain from Eqs. (14) and (15),

$$
\frac{p}{\sigma}=w=\frac{1}{4} \frac{\left(2 a-r_{-}-r_{+}\right)\left(r_{0}^{2}-a^{2}\right)}{\left(a-r_{-}\right)\left(a-r_{+}\right)\left[a+(1-2 n) r_{0}\right]}
$$

Observe that if the location of the wormhole throat is very large, i.e., if $a \rightarrow+\infty$, then $w \rightarrow-\frac{1}{2}$. On the other hand, if $a \rightarrow r_{+}$(from the right), then $\omega \rightarrow-\infty$. 


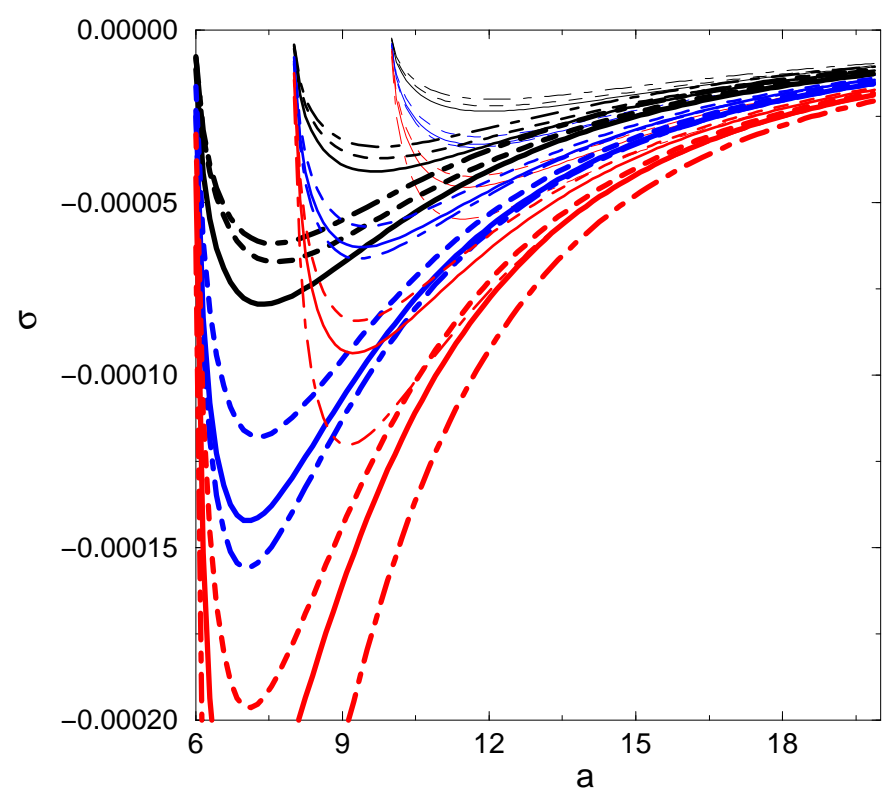

FIG. 2: Plot for $\sigma$ versus $a$. The description of the curves is the same as in FIG. 1]

So the distribution of matter in the shell is of the darkenergy type. Now, purely mathematicall speaking, if $a \rightarrow$ $\frac{1}{2}\left(r_{-}+r_{+}\right)$, then $p \rightarrow 0$. Since $\frac{1}{2}\left(r_{-}+r_{+}\right)<r_{+}$, however, such a dust shell is never found.

Our spacetime metric implies that the surface mass of this thin shell is given by $M_{\text {shell }}=4 \pi h(a) \sigma$. (For a static solution, we have $\dot{a}=0$ and $\ddot{a}=0$.) Thus

$$
\begin{aligned}
M_{\text {shell }}= & 2 \frac{\left[a+(1-2 n) r_{0}\right]}{\left(r_{0}^{2}-a^{2}\right)} \times \\
& \left(a-r_{0}\right)^{1-n}\left(a+r_{0}\right)^{n} \sqrt{\left(a-r_{-}\right)\left(a-r_{+}\right)} .
\end{aligned}
$$

Now observe that for $n=\frac{1}{2}$, the mass of the black hole in Eq. (10) is increasing with $r_{0}$. At the same time, for a fixed value of the throat radius $a$, the mass of the thin shell is decreasing with $r_{0}$, as long as $r_{0}$ remains much less than $a$.

\section{STABILITY}

Now we will focus our attention on the stability of the configuration under small perturbations around a static solution at $a_{0}$. The starting point is the definition of a potential, extended to our metric [Eq. (2)]. Rearranging Eq. (12), we obtain the thin shell's equation of motion

$$
\dot{a}^{2}+V(a)=0 .
$$

Here the potential $V(a)$ is defined as

$$
V(a)=f(a)-\left[\frac{4 \pi h(a) \sigma(a)}{h^{\prime}(a)}\right]^{2} .
$$

Expanding $V(a)$ around $a_{0}$, we obtain

$$
\begin{aligned}
V(a)= & V\left(a_{0}\right)+V^{\prime}\left(a_{0}\right)\left(a-a_{0}\right)+\frac{1}{2} V^{\prime \prime}\left(a_{0}\right)\left(a-a_{0}\right)^{2} \\
& +O\left[\left(a-a_{0}\right)^{3}\right],
\end{aligned}
$$

where the prime denotes the derivative with respect to $a$, assuming a static solution situated at $a_{0}$. Since we are linearizing around $a=a_{0}$, we must have $V\left(a_{0}\right)=0$ and $V^{\prime}\left(a_{0}\right)=0$. The configuration will then be in stable equilibrium if $V^{\prime \prime}\left(a_{0}\right)>0$.

To carry out this analysis, we start with the energy conservation equation. Using Eqs. (12) and (13), one can verify that

$$
\begin{aligned}
& \frac{d}{d \tau}(\sigma \mathcal{A})+ p \frac{d \mathcal{A}}{d \tau}= \\
&\left\{\left[h^{\prime}(a)\right]^{2}-2 h(a) h^{\prime \prime}(a)\right\} \frac{\dot{a} \sqrt{f(a)+\dot{a}^{2}}}{2 h(a)}
\end{aligned}
$$

where $\mathcal{A}=4 \pi h(a)$ by Eq. (2). The first term on the left side corresponds to a change in the throat's internal energy, while the second term corresponds to the work done by the throat's internal forces. According to Ref. [19], the right side represents a flux. From Eq. (27), we get

$$
\begin{aligned}
& \frac{d}{d a}[\sigma h(a)]+\mathcal{P} \frac{d}{d a} {[h(a)] } \\
&=-\left\{\left[h^{\prime}(a)\right]^{2}-2 h(a) h^{\prime \prime}(a)\right\} \frac{\sigma}{2 h^{\prime}(a)}
\end{aligned}
$$

and, finally,

$$
\begin{array}{r}
h(a) \sigma^{\prime}+h^{\prime}(a)(\sigma+p)+\left\{\left[h^{\prime}(a)\right]^{2}-2 h(a) h^{\prime \prime}(a)\right\} \frac{\sigma}{2 h^{\prime}(a)} \\
=0 .
\end{array}
$$

It is also shown in Ref. [19] that

$$
\begin{aligned}
& V^{\prime \prime}(a)=f^{\prime \prime}(a)+16 \pi^{2} \times \\
& \left\{\left[\frac{h(a)}{h^{\prime}(a)} \sigma^{\prime}(a)+\left(1-\frac{h(a) h^{\prime \prime}(a)}{\left[h^{\prime}(a)\right]^{2}}\right) \sigma(a)\right][\sigma(a)+2 p(a)]\right. \\
& \left.\quad+\frac{h(a)}{h^{\prime}(a)} \sigma(a)\left[\sigma^{\prime}(a)+2 p^{\prime}(a)\right]\right\} .
\end{aligned}
$$

Next, we define a parameter $\beta$, which is interpreted as the subluminal sound speed, by the relation

$$
\beta^{2}(\sigma)=\left.\frac{\partial p}{\partial \sigma}\right|_{\sigma}
$$

To do so, observe that

$$
\begin{aligned}
& \sigma^{\prime}(a)+2 p^{\prime}(a) \\
& =\sigma^{\prime}(a)\left[1+2 p^{\prime}(a) / \sigma^{\prime}(a)\right]=\sigma^{\prime}(a)\left(1+\beta^{2}\right)
\end{aligned}
$$


Using Eq. (28), we can now rewrite $V^{\prime \prime}(a)$ as follows:

$$
\begin{aligned}
& V^{\prime \prime}(a)=f^{\prime \prime}(a)-8 \pi^{2}\left\{[\sigma(a)+2 p(a)]^{2}\right. \\
& \left.+2 \sigma(a)\left[\left(\frac{3}{2}-\frac{h(a) h^{\prime \prime}(a)}{\left[h^{\prime}(a)\right]^{2}}\right) \sigma(a)+p(a)\right]\left(1+2 \beta^{2}\right)\right\} .
\end{aligned}
$$

At the static solution $a=a_{0}$, the conditions $V\left(a_{0}\right)=0$ and $V^{\prime}\left(a_{0}\right)=0$ are indeed met.

Now consider the stability criterion $V^{\prime \prime}\left(a_{0}\right)>0$ starting with Eq. (31): first let $V^{\prime \prime}\left(a_{0}\right)=0$ and solve for $\beta^{2}$. We then find that the graph of

$$
\beta^{2}=-\frac{1}{2}+\frac{f^{\prime \prime} / 8 \pi^{2}-(\sigma+2 p)^{2}}{4 \sigma\left[\left(\frac{3}{2}-\frac{h h^{\prime \prime}}{\left(h^{\prime}\right)^{2}}\right) \sigma+p\right]}
$$

has a single vertical asymptote (Fig. 3.) To the right of the asymptote,

$$
4 \sigma\left[\left(\frac{3}{2}-\frac{h h^{\prime \prime}}{\left(h^{\prime}\right)^{2}}\right) \sigma+p\right]>0,
$$

also determined graphically. Returning now to the inequality $V^{\prime \prime}\left(a_{0}\right)>0$, we therefore have at $a=a_{0}$

$$
\beta^{2}<-\frac{1}{2}+\frac{f^{\prime \prime} / 8 \pi^{2}-(\sigma+2 p)^{2}}{4 \sigma\left[\left(\frac{3}{2}-\frac{h h^{\prime \prime}}{\left(h^{\prime}\right)^{2}}\right) \sigma+p\right]} .
$$

So to the right of the asymptote, the region of stability is below the graph of Eq. (32), as shown in Fig. 3.

To the left of the asymptote, the sense of the inequality in (33) is reversed and we obtain at $a=a_{0}$

$$
\beta^{2}>-\frac{1}{2}+\frac{f^{\prime \prime} / 8 \pi^{2}-(\sigma+2 p)^{2}}{4 \sigma\left[\left(\frac{3}{2}-\frac{h h^{\prime \prime}}{\left(h^{\prime}\right)^{2}}\right) \sigma+p\right]} .
$$

So to the left of the asymptote, the region of stability is above the graph.

Fig. 3 does indeed show typical regions of stability using somewhat arbitrary values of the various parameters: $r_{0}=1, r_{-}=2, r_{+}=3$, and $n=1 / 2$. As noted above, the region is below the curve on the right and above the curve on the left. The sign change is determined by inequality (33). These results, including the graphs, are similar to those in Refs. [2] and 19] (dealing with Schwarzschild and dilaton thin-shell wormholes, respectively) in the sense that the regions do not correspond to any value in the interval $0<\beta^{2} \leq 1$. Since $\beta$ is ordinarily interpreted as the speed of sound, it is highly desirable to obtain a region for which $\beta^{2}<1$. This is indeed possible for our wormhole: if we choose $r=r_{-}$close to $r=r_{+}$, then we typically get a region of stability for $\beta^{2}<1$. For example, in Fig. $4, r_{0}=1$, $r_{-}=2, r_{+}=2.05$, and $n=0.8$. The closer $r_{-}$is to $r_{+}$, the more the region of stability extends below $\beta^{2}=1$.

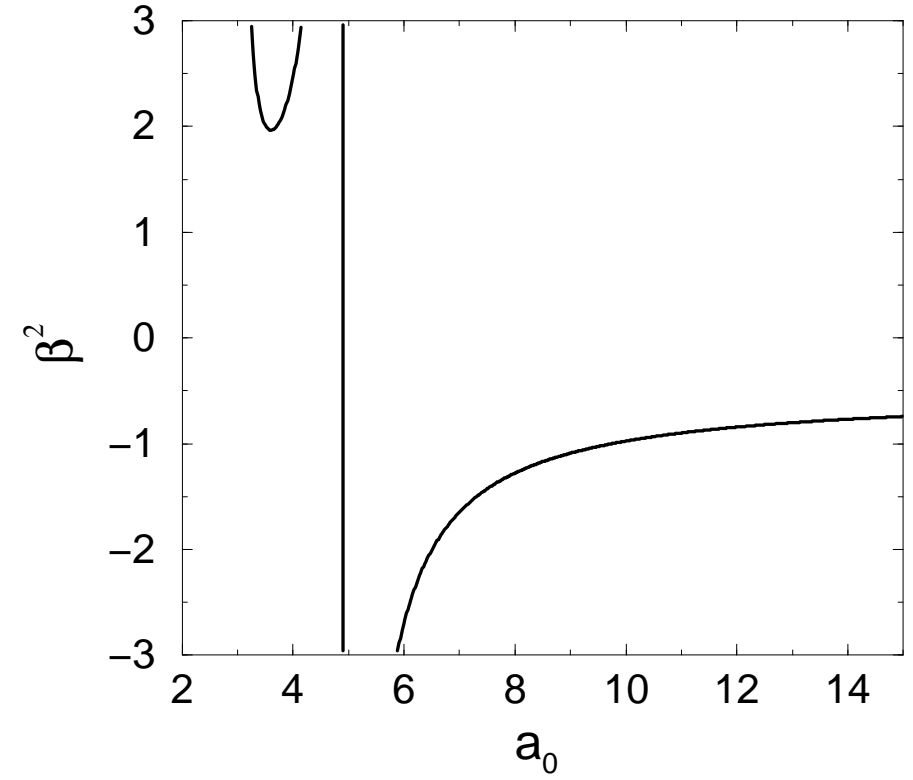

FIG. 3: Plot for $\beta^{2}$ versus $a_{0}$.

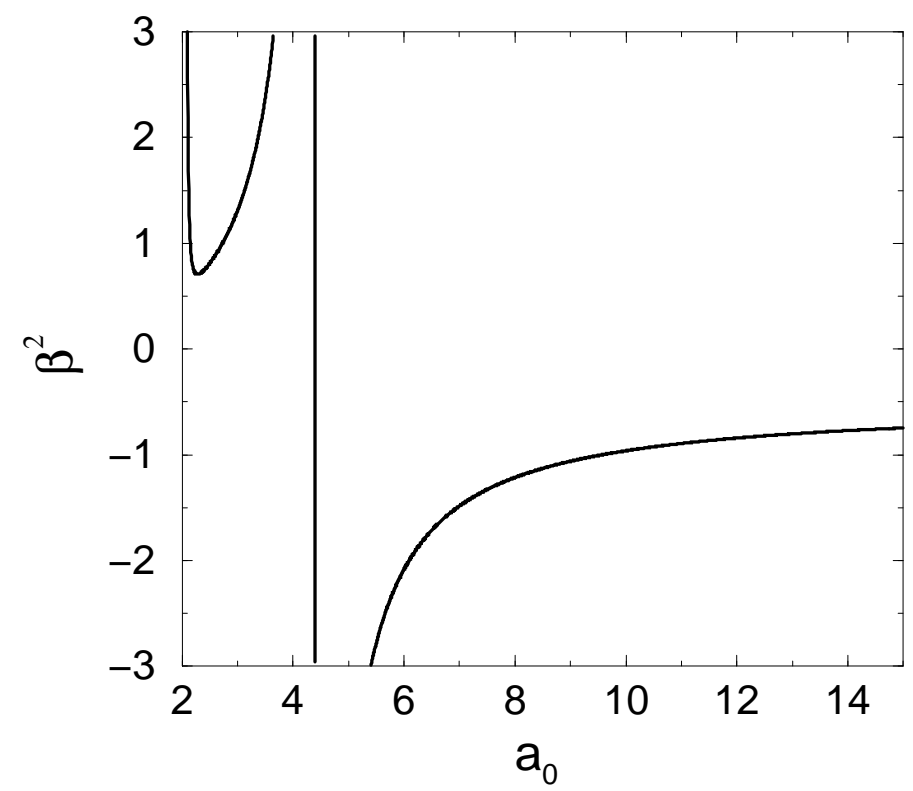

FIG. 4: All functions are the same. Some are cut off. See Fig. 3.

\section{CONCLUSION}

A new black-hole solution by Sur, et al., for EinsteinMaxwell scalar fields was inspired by low-energy string theory. This paper discusses a new thin-shell wormhole 
constructed by applying the cut-and-paste technique to two copies of such black holes. We analyzed various aspects of this wormhole, such as the amount of exotic matter required, the attractive or repulsive nature of the wormhole, and a possible equation of state for the thin shell. The stability analysis concentrated on the parameter $\beta$, normally interpreted as the speed of sound. It was found that whenever the two event horizons are close together, a stability region exists for some values of $\beta^{2}$ less than unity, unlike the the cases discussed in Refs. 2] and [19] for Schwarzschild and dilaton thin-shell wormholes, respectively.

\section{Acknowledgments}

AAU, FR and SR are thankful to Inter-University Centre for Astronomy and Astrophysics, Pune, India for providing Visiting Associateship under which a part of this work is carried out. FR and $\mathrm{ZH}$ are also grateful to UGC, Govt. of India and D.S. Kothari fellowship, for financial support.
[1] M. Visser, Nucl. Phys. B 328, (1989) 203.

[2] E. Poisson and M. Visser, Phys. Rev. D 52, (1995) 7318.

[3] F.S.N. Lobo and P. Crawford, Class. Quant. Grav. 21, (2004) 391.

[4] F.S.N. Lobo, Class. Quant. Grav. 21, (2004) 4811.

[5] E.F. Eiroa and G. Romero, Gen. Rel. Grav. 36, (2004) 651.

[6] E.F. Eiroa and C. Simeone, Phys. Rev. D 70, (2004) 044008.

[7] E.F. Eiroa and C. Simeone, Phys. Rev. D 71, (2005) 127501 .

[8] M. Thibeault, C. Simeone and E.F. Eiroa, Gen. Rel. Grav. 38, (2006) 1593

[9] F.S.N. Lobo, Phys. Rev. D 71, (2005) 124022.

[10] F. Rahaman et al., Gen. Rel. Grav. 38, (2006) 1687.

[11] E. Eiroa and C. Simeone, Phys. Rev. D 76, (2007) 024021.
[12] F. Rahaman et al., Int. J. Mod. Phys. D 16, (2007) 1669.

[13] F. Rahaman et al., Gen. Rel. Grav. 39, (2007) 945.

[14] F. Rahaman et al., Chin. J. Phys. 45, (2007) 518.

[15] J. P. S. Lemos and F.S.N. Lobo, Phys. Rev. D 78, (2008) 044030.

[16] M. G. Richarte and C. Simeone, Phys. Rev. D 76, (2007) 087502.

[17] F. Rahaman et al., Acta Phys. Polon. B 40, (2009) 1575.

[18] F. Rahaman et al., Mod. Phys. Lett. A 24, (2009) 53.

[19] E.F. Eiroa, Phys. Rev. D 78, (2008) 024018.

[20] E.F. Eiroa, M.G. Richarte, and C. Simeone, Phys. Lett. A 373 (2008) 1.

[21] S. Sur, S. Das, and S. SenGupta, JHEP 0510, (2005) 064.

[22] T.A. Roman, Phys. Rev. D 53, (1993) 5496. 